\title{
Corrigendum: Artemisinin-resistant Plasmodium falciparum clinical isolates can infect diverse mosquito vectors of Southeast Asia and Africa
}

\author{
Brandyce St. Laurent, Becky Miller, Timothy A. Burton, Chanaki Amaratunga, Sary Men, Siv Sovannaroth, \\ Michael P. Fay, Olivo Miotto, Robert W. Gwadz, Jennifer M. Anderson \& Rick M. Fairhurst
}

Nature Communications 6:8614 doi: 10.1038/ncomms9614 (2015); Published 20 Oct 2015; Updated 8 Jan 2016

In the first paragraph of the Results section in this Article, the An. gambiae G3 line is incorrectly stated to have been artificially selected for permissiveness to infection by multiple P. falciparum strains. The following sentence is correct: 'These latter two lines are highly permissive to infection by multiple P. falciparum strains, a phenotype for which An. stephensi Nijmegen was artificially selected' (Feldmann and Ponnudurai). In addition, in the second paragraph of the Discussion section, a reference to a recent report that the $P f s 47$ gene mediates the differential infectivity of three $P$. falciparum strains incorrectly states that this was demonstrated in the An. stephensi Nijmegen and An. gambiae G3 lines. This should have read: 'Although elucidating the molecular basis of this finding requires further investigation, we have excluded a possible role for polymorphism in the Pfs47 gene (PF3D7_1346800), which was recently shown to mediate the differential infectivity of the NF54, GB4 and 7G8 P. falciparum strains in the An. gambiae G3-L35 line'.

Feldmann, A. M. \& Ponnudurai, T. Selection of Anopheles stephensi for refractoriness and susceptibility to Plasmodium falciparum. Med. Vet. Entomol. 3, 41-52 (1989). 